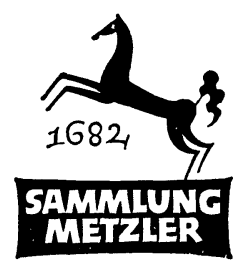

REALIEN ZUR LITERATUR

ABT. E:

POETIK

- 
FRITZ SCHLAWE

\section{Neudeutsche Metrik}

MCMLXXII

J.B.METZLERSCHE VERLAGSBUCHHANDLUNG

STUTTGART 
ISBN 978-3-476-10112-9

ISBN 978-3-476-03832-6 (eBook)

DOI 10.1007/978-3-476-03832-6

M I I 2

(C) Springer-Verlag GmbH Deutschland 1972

Ursprünglich erschienen bei J.B. Metzlersche Verlagsbuchhandlung und Carl Ernst Poeschel Verlag GmbH in Stuttgart 1972 


\section{VORWORT}

Der folgende Versuch einer Einführung in die neuere deutsche Metrik kann natürlich nur die Hauptpunkte berühren und einen Überblick geben. Erschwert war die Aufgabe durch die bekannte Tatsache, daß es keine allgemein anerkannten metrischen Grundlagen gibt. Die vorliegende Arbeit vertritt eine - wenn man so sagen darf - inhaltsbezogene Metrik: sie beschäftigt sich mit den metrischen Elementen im Hinblick auf deren sprachgegebene rhythmische Prägungen; weil die metrischen Elemente erst in der Umprägung ihrer jeweils individuellen sprachlichen Verwirklichung zu ästhetischen Phänomenen werden.

Der enge Rahmen bietet keinen Raum für eine Geschichte der Metrik als Wissenschaft; eine Übersicht gibt ULRICH Pretzel (iDt. Philologie im Aufriß<, III, ${ }^{2}$ I962, Sp. 23592367,252 Iff.), ältere Literatur im Überfluß JАСОв MinoR ("Neuhochdt. Metrik«, ${ }^{2}$ I902, S. 5 I I-537). Auch die Literaturangaben werden hier beschränkt, weil sie bei Pretzel ausführlich und leicht zugänglich sind (Sp. 252 I-2546); nur die neuen, dort nicht verzeichneten Arbeiten werden möglichst vollständig genannt. Reichhaltige Bibliographie findet sich auch in den Artikeln des >Reallexikons` (s. u. S. 6). Für die ältere deutsche Metrik darf auf das Parallel-Bändchen »Altdeutsche Metrik» der 'Sammlung Metzler` von Werner Hoffmann verwiesen werden; vgl. ferner die »Germanische Verskunst« von KLAUS v. SEE, SM Bd 67, I967. Allgemeinere Literatur, die man über die bibliographischen Hilfsmittel - Arnold, KöRner, HANSEL, EPPELSHeImer usw. - leicht feststellen kann, wird grundsätzlich nicht aufgeführt.

Um Indemnität habe ich zu bitten wegen ungewöhnlich häufiger Zitate im theoretischen Teil; sie schienen mir wegen des Fehlens einer communis opinio an vielen Stellen wünschenswert, um dem Leser eigenes Urteil zu erleichtern. Auch wird man verstehen, daß ich einige Dinge, über die man Lexika nachschlagen kann, kürzer behandelt habe zugunsten anderer, bei denen das nicht ohne weiteres möglich ist.

Tübingen/Cincinnati, März 1972

F. S. 


\section{INHALT}

I. Einführung . . . . . . . . . . . . . . . . I

I.r. Aufgabenbereich der Metrik . . . . . . . . I

I.2. Geschichte der Metrik . . . . . . . . . 2 I.2.r. Vorstufen ... . . . . . . . . 2

I.2.2. Endstufe . . . . . . . . . . . . 4

I.3. Grundfragen der Metrik . . . . . . . . . 7

I.3.r. Metrum-Vers. . . . . . . . . . . . 7

I.3.2. Metrum-Rhythmus . . . . . . . . . 8

I.3.3. Metrum-Sprache . . . . . . . . . . 9

I.3.4. Metrum-Schallform . . . . . . . . . . II

I.3.5. Metrische Deskription . . . . . . . . . II

2. Grundbegriffe. . . . . . . . . . . . . . . I3

2.I. Der metrische Rahmen . . . . . . . . . . . I3

2.I.I. Hauptphänomene . . . . . . . . . . I I3

2.r.r.I. Hebung und Senkung ....... . I3

2.r.r.2. Akzent . . . . . . . . . . $\mathrm{I} 6$

2.r.2. Konkrete Einheiten . . . . . . . . . . . I9

2.r.2.r. Versfuß . . . . . . . . . . I9

2.1.2.2. Verszeile .......... . 20

2.r.2.3. Strophe. . . . . . . . . . $2 \mathrm{I}$

2.I.3. Das Metametrische . . . . . . . 36

2.2. Die übermetrische Prägung . . . . . . . 38

2.2.I. Hauptphänomene . . . . . . . . 38

2.2.r.r. Takt . . . . . . . . . . 38

2.2.1.2. Rhythmus . . . . . . . . . 42

2.2.2. Konkrete Einheiten . . . . . . . . . . . 47

2.2.2.I. Wortfuß . . . . . . . . . 47

2.2.2.2. Kolon . . . . . . . . . . . . 49

2.2.2.3. Satzeinheit . . . . . . . . 54 
3. Formen. . . . . . . . . . . . . . . . 55

3.r. Versmaße der deutschen Dichtung . . . . . . 55

3.r.x. Strophische $\mathrm{Maße}$. . . . . . . . 55

3.r.r.r. Jamben . . . . . . . . . 55

3.r.r.2. Trochäen. . . . . . . . . 60

3.I.I.3. Alternierende Langzeilen . . . . . . . 62

3.r.r.4. Daktylen (Doppelsenkungen) . . . . . 62

3.r.2. Nichtstrophische Maße . . . . . . . . $6_{3}$

3.I.2.r. Madrigalverse. . . . . . . . $6_{3}$

3.r.2.2. Freie Verse . . . . . . . . . . . 64

3.r.2.3. Knittelverse . . . . . . . . 65

3.I.2.4. Vers irréguliers . . . . . . . . 67

3.I.2.5. Freie Rhythmen . . . . . . . . 67

3.1.2.6. Hexameter/Pentameter . . . . . . . . 69

3.I.2.7. Trimeter......... $7^{\text {I }}$

3.2. Strophenformen der deutschen Lyrik . . . . . . 72

3.2.r. Antike Formen . . . . . . . . . . 72

3.2.I.I. Oden (alkäische, sapphische, asklepiadeische Strophe) . . . . . . . 72

3.2.1.2. Distichon . . . . . . . . . 76

3.2.r.3. Neuantikes . . . . . . . . 77

3.2.2. Orientalische Formen . . . . . . . . . . . 77

3.2.2.I. Arabische Formen (Ghazel, Kassidé; Makame) . . . . . . . . . . . . 78

3.2.2.2. Persische Form (Rubâi) . . . . . . . 79

3.2.3. Romanische Formen . . . . . . . . 79

3.2.3.I. Italienische Strophen (Sonett, Stanze, Terzine, Sestine, Triolett, Ritornell, Madrigal, Kanzone) . . . . . . . 80

3.2.3.2. Spanische Strophen (Dezime, Kanzion)

3.2.3.3. Französische Strophen (Quatrain, Rondeau) .......... 86

3.3. Quellen . . . . . . . . . . 87

3.3.r. Das Kirchenlied . . . . . . . . . . . . . . 88

3.3.2. Das Volkslied . . . . . . . . . . . 89

4. Anhang . . . . . . . . . . . . . 92

5. Register ................ I04 